\title{
PREOPERATIVE SERUM LEVELS OF SOLUBLE ENDOGLIN FOR PREDICTION OF RECURRENCE IN STAGE III COLORECTAL CANCER PATIENTS
}

\author{
Ismail Gomceli', Mesut Tez ${ }^{2}$, Erdal B. Bostanci ${ }^{1}$, Nesrin Turhan ${ }^{3}$, Ahu S. Kemik', Musa Akoglu ${ }^{1}$
}

Turkiye Yuksek Ihtisas Education and Research Hospital, Department of Gastroenterologic Surgery, Ankara, Turkey ${ }^{1}$; Ankara Numune Education and Research Hospital, Department of General Surgery, Ankara, Turkey2; Turkiye Yuksek Ihtisas Education and Research Hospital Department of Pathology, Ankara, Turkey ${ }^{3}$; Istanbul University Cerrahpasa Medical Faculty, Department of Biochemistry, Istanbul, Turkey ${ }^{4}$

Summary: Background: To evaluate the clinical usefulness of serum levels of soluble form of endoglin in stage III colorectal adenocarcinomas (CRC) patients for detection of recurrence. Methods: The case-control study consisted of 80 stage III CRC patients who underwent surgery with curative intent and 70 age-and sex-matched healthy volunteers. Serum levels of soluble form of endoglin (sol-end) were measured in both groups. Also, predictive factors of recurrence were evaluated using multivariate analyses. Results: Serum levels of sol-end in stage III CRC patients were significantly higher than those in controls. There was not a significant association between serum levels of sol-end and clinicopathological features in CRC patients. Multivariate regression analysis showed the LNR (hazard ratio, 2.54; $95 \%$ CI, $1.46-4.34 ; \mathrm{p}<0.001)$, to be significant independent factors to estimate local recurrence in stage III CRC patients. Conclusion: Preoperative serum levels of sol-end do not seem useful as a marker for detection of recurrence in stage III CRC patients.

Key words: Colon; Rectum; Cancer; Endoglin

\section{Introduction}

Colorectal cancer (CRC) is one of the most common malignancies and the second leading cause of death from cancer in Europe and North America (1). While earlystage CRC is associated with an excellent 5-year survival rate $(90 \%$ for localized disease), approximately $20 \%$ of patients present with metastatic disease, and many patients diagnosed with stage II or III cancer will experience a recurrence and develop distant metastases (2).

At present, established clinico-pathological criteria are used to estimate risks of recurrence in stage II and III disease, and this is routinely used in the selection of patients for adjuvant systemic therapy following surgical resection. The clinical outcome of patients who receive such adjuvant treatment can, however, vary widely, when additional molecular factors are taken into consideration. Identification of novel prognostic markers is, therefore, vital in improving the prognosis of this disease (3).

One of the recently described substances important for angiogenesis is endoglin. Endoglin, also known as CD105, is a receptor for transforming growth factor- $\beta 1$ molecule, which binds preferentially to the activated endothelial cells that participate in tumor angiogenesis, with weak or negative expression in vascular endothelium of normal tissues. Endoglin is induced by hypoxia. Therefore it is very useful for assessment of neo-angiogenesis of malignant neoplasms (4-6). Many reports indicate that endoglin assessed immunohistochemically in colorectal cancer correlates not only with tumor microvessel density, but also with survival. It has been also reported as a valuable parameter predicting patients having an increased risk of developing metastatic disease. Endoglin is expressed not only on cell surface, since its soluble form (sol-end) can be detected also in blood (4-7). A few of studies evaluated the clinical significance of elevated solend levels in colorectal cancer patients (7).

In the current study, we assessed a possible role of the preoperative serum levels of sol-end as a potent predictor of recurrence in stage III CRC patients.

\section{Materials and Methods}

Eighty patients with histologically confirmed stage III CRC patients according to TNM staging system were included in this study. There were 47 men and 33 women with a median age of 62 (range, 33-87) years. Tumor staging was based on clinical information, radiologic reports (chest radiography, abdominal ultrasonography and computerized tomography), operative findings, and pathology reports. Patients were followed prospectively and the date and cause of death was recorded. The median follow-up of the patients was 18 (range, 3-30) months. 
Control subjects consisted of 70 age-and sex-matched healthy volunteers (median age 55 (range, 43-68) years; 38 men and 32 women)

The clinicopathologic parameters studied for prognostic value were tumor localization, histopathologic grade, tumor size $(4 \mathrm{~cm}<, 4 \mathrm{~cm} \geq)$, the ratio of metastatic to retrieved lymph nodes (LNR), perineural invasion, vascular invasion and serum CEA level.

All patients provided informed consent before inclusion in the study.

This study has been approved by the institutional review board of Turkiye Yuksek Ihtisas Education and Research Hospital. The regional ethics committee approved the project and written informed consent was obtained from all patients and controls before their inclusion.

Control blood samples were obtained from 60 individuals who visited health examination clinics with minimal gastritis or normal appearance of the gastric mucosa on endoscopic examination. Blood samples were collected before malignancies were treated surgically. Samples were allowed to clot and serum was obtained after sample centrifugation. Serum samples were stored frozen at $-20^{\circ} \mathrm{C}$. Repeated thawing and freezing of samples was avoided. Soluble form of endoglin in serum was assessed by sandwich ELISA using diagnostic kit manufactured by R\&D Systems (USA). The standard range of this kit is from 0.15 to $10 \mathrm{ng} / \mathrm{ml}$ and the minimum detectable dose is $0.007 \mathrm{ng} / \mathrm{ml}(8)$.

\section{Statistical Analysis}

Recurrence was defined as any locoregional recurrence (tumor tissue at the primary site of resection, either intra- or extraluminal) and/or any distant metastasis (distant spread to other organs), alone or in combination.

Statistical analysis was performed using the SPSS statistical software package. The demographic characteristics of patients and controls were compared using the $\chi^{2}$ square test and Student's t test. Shapiro-Wilk test was used for assessing normality. Accordingly, all data are presented as median value (interquartile range), with nonparametric analyses being employed to assess differences. The Kruskal-Wallis analysis of variance (ANOVA) and the Mann-Whitney U test were used to evaluate differences between multiple groups, and unpaired observations, respectively. Correlations were evaluated using the Spearman rank test. Multivariable Cox proportional hazards regression analysis addressed the time to recurrence and relied on the established clinical predictors. Significance was presumed at $\mathrm{p}<0.05$.

\section{Results}

\section{Serum levels of sol-end in healthy controls}

Serum levels of sol-end were detectable in all healthy controls. The median serum level of sol-end was 12.75 (11.5-14.95) ng/mL.
There was no significant difference in serum levels of sol-end between males and females (12.65 (11.5-14.90) $\mathrm{ng} / \mathrm{mL}$ versus 13.6 (11.6-15.7) $\mathrm{ng} / \mathrm{mL}$, respectively; $p=$ $0.493)$. No correlation was found between serum levels of sol-end and age $(r=0.23 ; p=0.53)$.

\section{Serum levels of sol-end in stage III CRC patients}

The median serum level of sol-end in stage III CRC patients was 64 (52.30-74.50) ng/mL and significantly higher than those in controls $(\mathrm{p}<0.001)$. There was no significant difference between males and females regarding serum levels of sol-end $(63.20(53.50-81) \mathrm{ng} / \mathrm{mL}$ versus 64.60 $(51.50-70.90) \mathrm{ng} / \mathrm{mL}$, respectively; $p=0.61$ ) nor there was any correlation between serum levels of sol-end, age $(r=$ $0.052 ; p=0.65)$ and serum CEA levels $(\mathrm{r}=0.141 ; \mathrm{p}=0.239)$. There were no significant associations between serum levels of sol-end and, histopathologic grade, tumor size, LNR, perineural invasion and vascular invasion (Table 1).

Tab. 1: The relationship between preoperative serum levels of sol-end and pathologic variables in stage III CRC patients.

\begin{tabular}{|c|c|c|c|}
\hline \multirow{2}{*}{ Variables } & & & \\
\hline & $\mathrm{N}$ & Endoglin $\mathrm{ng} / \mathrm{mL}$ & Significance \\
\hline \multicolumn{4}{|l|}{ Tumor localization } \\
\hline $\begin{array}{l}\text { Right colon(Cecum, } \\
\text { Ascending colon, Trans- } \\
\text { verse colon) }\end{array}$ & 24 & $72.40(59.00-88.20)$ & \multirow{4}{*}{ NS } \\
\hline $\begin{array}{l}\text { Left colon( Descending } \\
\text { colon, Sigmoid colon) }\end{array}$ & 19 & $78.30(69.20-90.40)$ & \\
\hline Rectum & 37 & $71.30(59.30-86.30)$ & \\
\hline Differentiation & & & \\
\hline Well & 28 & $69.00(54.00-77.55)$ & \multirow{4}{*}{ NS } \\
\hline Moderate & 40 & $59.40(50.90-74.80)$ & \\
\hline Poor & 12 & $67.80(61.90-73.70)$ & \\
\hline Tumor diameter & & & \\
\hline$<4 \mathrm{~cm}$ & 7 & $60.30(52.30-74.50)$ & \multirow[t]{2}{*}{ NS } \\
\hline$\geq 4 \mathrm{~cm}$ & 77 & $65.05(52.90-75.55)$ & \\
\hline \multicolumn{3}{|l|}{ Perineural invasion } & \multirow{3}{*}{ NS } \\
\hline Positive & 24 & $65.20(54.50-77.40)$ & \\
\hline Negative & 53 & $59.20(50.80-74.25)$ & \\
\hline
\end{tabular}




\begin{tabular}{|c|c|c|c|}
\hline \multirow{2}{*}{ Variables } & & & \multirow[b]{2}{*}{ Significance } \\
\hline & $\mathrm{N}$ & Endoglin ng/mL & \\
\hline Unknown & 3 & & \\
\hline \multicolumn{4}{|c|}{ Vascular invasion } \\
\hline Positive & 40 & $59.30(50.70-74.80)$ & \multirow{2}{*}{ NS } \\
\hline Negative & 40 & $65.70(54.30-73.40)$ & \\
\hline \multicolumn{4}{|c|}{ Lymph node ratio } \\
\hline$<.16$ & 62 & $64(50.90-80.30)$ & \multirow{2}{*}{ NS } \\
\hline $.16<$ & 18 & $62.15(53.40-70.45)$ & \\
\hline
\end{tabular}

Values are median (interquartile range) NS: Non-significant

\section{Serum levels of sol-end, and local recurrence}

Eighteen patients had local recurrence of stage III CRC during the follow-up period. There was not significant difference between the patients with local recurrence and non local recurrence regarding serum levels of sol-end (64 (52.90-74.65) ng/mL versus 62.15 (52.30-71.40) ng/mL, respectively, $p=0.7$ )

Univariate analysis showed, LNR $(\mathrm{p}=0.19)$, differentiation $(p<0.01)$, tumor diameter $(p=0.17)$, high CEA level $(p=0.036)$, perineural $(p=0.034)$ and perivascular $(\mathrm{p}=0.02)$ invasion to be significant factors affecting local recurrence. Multivariate regression analysis showed the LNR (hazard ratio, 2.54; 95\% CI, 1.46-4.34; $\mathrm{p}<0.001$ ), to be significant independent factors for estimation of local recurrence.

\section{Discussion}

Our study shows a marked difference between preoperative serum levels of sol-end in stage III CRC patients when compared with age and sex-matched controls, without an association between these levels and clinicopathologic features.

Endoglin has been reported to be an indicator of poor prognosis in many types of solid neoplasia such as: breast carcinoma, cervical cancer, endometrial carcinoma, gastric carcinoma, melanoma, some testicular tumors, non-small cell lung cancer, prostate cancer, renal cell carcinoma and squamous cell carcinoma (9).

Saad et al immunostaed surgical specimens from 150 patients with resected colorectal carcinomas for endoglin. In this study, endoglin, by staining higher numbers of the proliferating vessels in colon carcinoma, is a more specific and sensitive marker for tumor angiogenesis than the commonly used panendothelial markers. Endoglin staining also showed prognostic significance with positive correlation with angiolymphatic invasion and metastases to lymph nodes and liver (10). Similarly, Akagi et al. investigated 54 cases of colorectal adenomas and 20 cases of carcinomas using monoclonal antibodies CD105. They found that assessing neovascularization with CD105 in the process of colorectal cancer development may be a valuable marker for predicting the risk of colorectal cancer development (11).

Soluble endoglin has been reported to rise in plasma of breast cancer patients who developed metastases as compared to both normal controls and cancer patients without distant spread (12). Elevated serum endoglin was associated with distant metastases not only in breast, but also in colorectal cancer and in other solid tumors (13). Also Li et al. (14) using an indirect

sandwich ELISA described a correlation between plasma endoglin and Dukes' stages.

Myoeliwiec et al showed that endoglin assessment in plasma does not seem useful as a marker of colorectal cancer. However, that it might be helpful in selecting patients with metastatic disease (7).

Several reports have suggested that the postoperative serum CEA level is a useful marker of recurrence after colorectal surgery. Moreover, it can be measured cheaply and easily. Monitoring of the postoperative CEA level is thus commonly used in the follow-up of CRC patients. However, there has been some controversy about the significance of the preoperative serum CEA level as a predictive factor of recurrence (15). In this study, we found that LNR is an independent predictive factor for overall survival. The cut-off value of 0.16 for LNR derived from previous studies. Our data complement the conclusions of other previously published studies. Chen et al demonstrated that metastatic LNR independently estimates survival in Stage III colon cancer, irrespective of number of nodes examined (16). Berger et al 13 analyzed data from the Inter-group Trial 0089 using slightly different cutoffs for LNR $(5 \%$, $5-20 \%, 20-40 \%$, and $>40 \%$ ) finding LNR was a significant prognostic factor (17). Lee and colleagues also demonstrated that the ratio-based LN staging can be considered a simple and reproducible modality to assess prognosis of patients who underwent potentially curative radical resection for LN-positive colon cancer (18).

A weak point in this study is that we have not examined the association between tissue endoglin expression and serum levels in the same patients.

In conclusion, this study demonstrated that serum levels of sol-end were higher in stage III CRC patients than normal controls. These levels did not correlate with clinicopathologic parameters and serum levels of sol-end are not a good marker for prediction of local recurrence in stage III CRC patients.

\section{References}

\footnotetext{
1. Ferlay J, Autier P, Boniol M, Heanue M, Colombet M, Boyle P. Estimates of the cancer incidence and mortality in Europe in 2006. Ann Oncol. 2007; 18: 581-592.

2. Meyerhardt JA, Mayer RJ. Systemic therapy for colorectal cancer. N Engl J Med 2005; 352: 476-487.
} 
3. Allegra CJ, Paik S, Colangelo LH, Parr AL, Kirsch I, Kim G, Klein P, Johnston PG, Wolmark N, Wieand HS. Prognostic value of thymidylate synthase, Ki-67, and p53 in patients with Dukes' B and C colon cancer: a National Cancer Institute-National Surgical Adjuvant Breast and Bowel Project collaborative study. J Clin Oncol. 2003; 21: 241-50.

4. Mysliwiec P, Pawlak K, Bandurski R, Kedra B. Soluble angiogenesis markers in gastric tumor patients. Folia Histochem Cytobiol. 2009; 47: 81-6.

5. Nikiteas NI, Tzanakis N, Theodoropoulos G, Atsaves V, Christoni Z, Karakitso $\mathrm{P}$, et al.Vascular endothelial growth factor and endoglin (CD-105) in gastric cancer.Gastric Cancer. 2007; 10: 12-7.

6. Takahashi N, Kawanishi-Tabata R, Haba A, Tabata M, Haruta Y, Tsai H, et al Association of serum endoglin with metastasis in patients with colorectal, breast, and other solid tumors, and suppressive effect of chemotherapy on the serum endoglin. Clin Cancer Res. 2001; 7: 524-32.

7. Myśliwiec P, Pawlak K, Kukliński A, Kedra B. Combined perioperative plasma endoglin and VEGF-A assessment in colorectal cancer patients. Folia Histochem Cytobiol. 2008; 46: 487-92.

8. Haruta Y, Seon BK. Distinct human leukemia-associated cell surface glycoprotein GP160 defined by monoclonal antibody SN6. Proc Natl Acad Sci U.S.A 1986; 83: 7898-902.

9. Fonsatti E, Maio M. Highlights on endoglin (CD105): from basic findings towards clinical applications in human cancer. J Transl Med. 2004; 2: 18.

10. Saad RS, Liu YL, Nathan G, Celebrezze J, Medich D, Silverman JF. Endoglin (CD105) and vascular endothelial growth factor as prognostic markers in colorectal cancer. Mod Pathol. 2004; 17: 197-203.

11. Akagi K, Ikeda Y, Sumiyoshi Y, Kimura Y, Kinoshita J, Miyazaki M, et al. Estimation of angiogenesis with anti-CD105 immunostaining in the process of colorectal cancer development. Surgery. 2002; 131: S109-113.
12. Li C, Guo B, Wilson P, Stewart A, Byrne G, Bundred N, et al. Plasma levels of soluble CD105 correlate with metastasis in patients with breast cancer. Int J Cancer. 2000; 89: 122-126.

13. Takahashi N, Kawanishi-Tabata R, Haba A, Tabata M, Haruta Y, Tsai H, et al. Association of serum endoglin with metastasis in patients with colorectal, breast, and other solid tumors, and suppressive effect of chemotherapy on the serum endoglin. Clin Cancer Res. 2001; 7: 524-532.

14. Li C, Gardy R, Seon BK, Duff SE, Abdalla S, Renehan A, et al. Both high intratumoral microvessel density determined using CD105 antibody and elevated plasma levels of CD105 in colorectal cancer patients correlate with poor prognosis. Br J Cancer. 2003; 88: 1424-1431.

15. Takagawa R, Fujii S, Ohta M, Nagano Y, Kunisaki C, Yamagishi S, Osada S, Ichikawa Y, Shimada H. Preoperative serum carcinoembryonic antigen level as a predictive factor of recurrence after curative resection of colorectal cancer. Ann Surg Oncol. 2008; 15: 3433-9.

16. Chen SL, Steele SR, Eberhardt J, Zhu K, Bilchik A, Stojadinovic A. Lymph node ratio as a quality and prognostic indicator in stage III colon cancer. Ann Surg. $2011 ; 253: 82-7$

17. Berger AC, Sigurdson ER, LeVoyer T, et al. Colon cancer survival is associated with decreasing ratio of metastatic to examined lymph nodes. J Clin Oncol. 2000; 23: 8706-8712.

18. Lee HY, Choi HJ, Park KJ, et al. Prognostic significance of metastatic lymph node ratio in node-positive colon carcinoma. Ann Surg Oncol. 2007; 14: 1712-1717.

Received: 20/10/2011

Accepted in revised form: 09/05/2012

\section{Corresponding author:}

Mesud Tez, 5.cadde 10/3 Bahçelievler,06500 Ankara/TURKEY; e-mail:mesuttez@yahoo.com 